\title{
UIT: Ultraviolet Observations of the Small Magellanic Cloud
}

\author{
Robert H. Cornett ${ }^{1}$ Michael R. Greason ${ }^{1}$ Jesse K. Hill ${ }^{1}$ \\ Joel Wm. Parker ${ }^{2}$ William H. Waller ${ }^{1}$ \\ Ralph C. Bohlin ${ }^{3}$ Kwang-Peng Cheng ${ }^{4}$ Susan G. Neff $f^{5}$ Robert W. O'Connell ${ }^{6}$ \\ Morton S. Roberts ${ }^{7}$ Andrew M. Smith ${ }^{5}$ and Theodore P. Stecher ${ }^{5}$
}

\begin{abstract}
A mosaic of four UIT far-UV (FUV) $\left(\lambda_{e f f}=1620 \AA\right)$ images, with derived stellar and HII region photometry, is presented for most of the Bar of the SMC. The UV morphology of the SMC's Bar shows that recent star formation there has left striking features including: a) four concentrations of UV-bright stars spread from northeast to southwest at nearly equal $(\sim 30 \operatorname{arcmin}=0.5 \mathrm{kpc})$ spacings; b) one of the concentrations, near DEM 55, comprises a well-defined 8 -arcmin diameter ring surrounded by a larger $\mathrm{H} \alpha$ ring, suggestive of sequential star formation.

FUV PSF photometry is obtained for 11,306 stars in the FUV images, resulting in magnitudes $\mathrm{m}(162)$. We present a FUV luminosity function for the SMC bar, complete to $\mathrm{m}(162) \sim 14.5$. Detected objects are well correlated with other SMC Population I material; of $711 \mathrm{H} \alpha$ emission-line stars and small nebulae within the UIT fields of view, 520 are identified with FUV sources. The FUV photometry is compared with available ground-based catalogs of supergiants, yielding 191 detections of 195 supergiants with spectral type earlier than F0 in the UIT fields. The $(\mathrm{m}(162)-\mathrm{V})$ color for supergiants is a sensitive measure of spectral type. The bluest observed colors for each type agree well with colors computed from unreddened Galactic spectral atlas stars
\end{abstract}

\footnotetext{
${ }^{1}$ Hughes STX Corporation, Code 681, Goddard Space Flight Center, Greenbelt MD 20771

${ }^{2}$ Southwest Research Institute, Boulder CO

${ }^{3}$ Space Telescope Science Institute, Homewood Campus, Baltimore, MD 21218

${ }^{4}$ Department of Physics, California State University, Fullerton, CA 92634

${ }^{5}$ Laboratory for Astronomy and Solar Physics, Code 680, Goddard Space Flight Center, Greenbelt MD 20771

${ }^{6}$ University of Virginia, Astronomy Department, P.O. Box 3818, Charlottesville, VA 22903

${ }^{7}$ National Radio Astronomy Observatory, Edgemont Rd., Charlottesville, VA 22903
} 
for types earlier than about A0; for later spectral types the observed SMC stars range significantly bluer, as predicted by comparison of low-metallicity and Galactic-composition models. Redder colors for some stars of all spectral types are attributed to the strong FUV extinction arising from even small amounts of SMC dust. Internal SMC reddenings are determined for all catalog stars. All stars with $\mathrm{E}(\mathrm{B}-\mathrm{V})>0.15$ are within regions of visible $\mathrm{H} \alpha$ emission.

FUV photometry for $42 \mathrm{H} \alpha$-selected HII regions in the SMC Bar is obtained for stars and for total emission (as measured in HII-region-sized apertures). The flux-weighted average ratio of total to stellar FUV flux is 2.15; consideration of the stellar FUV luminosity function indicates that most of the excess total flux is due to scattered FUV radiation, rather than stars fainter than $\mathrm{m}(162)=14.5$. Both stellar and total emission are well correlated with $\mathrm{H} \alpha$ fluxes measured by Kennicutt and Hodge (1986; hereafter KH), yielding $\mathrm{FUV} / \mathrm{H} \alpha$ flux ratios that are consistent with models of SMC metallicity, ages from 1-5 Myr, and moderate $(\mathrm{E}(\mathrm{B}-\mathrm{V})=0.0-0.1 \mathrm{mag})$ internal SMC extinction.

\section{Introduction}

UV observations from above the earth's atmosphere are vital for understanding the Population I properties of metal-poor galaxies such as the Small Magellanic Cloud (SMC). Optical-band studies of the SMC have shown that this nearby dwarf irregular galaxy provides a unique laboratory for investigating stellar and interstellar evolution at "primitive" compositions, compared to the Galaxy. Many effects of composition differences appear best, or only, in the UV. Line blanketing strongly affects UV colors; similarly, the steep SMC extinction curve $\left(\mathrm{A}_{162} / E(B-V) \sim 16\right)$, widely thought to be due to abundances in SMC dust, is "extreme" only in the UV. Moreover, because the energy distributions of hot stars peak at short wavelengths, FUV photometry is more effective than optical-band photometry in determining the temperatures of such stars. Here, we present initial results from a UV imaging study of the the SMC, based on a mosaic of four far-UV images that were obtained by the Ultraviolet Imaging Telescope (UIT) during the Astro-1 and Astro-2 missions in late 1990 and early 1995.

\section{Observations and Data Reduction}

UIT observed a total of four 40-arcmin diameter fields nearly completely covering the SMC Bar at an angular resolution of $\sim 3$ arcsec- better than 75 times the resolution of 
previous UV imaging studies of the SMC (Okumura 1993). A description of individual exposures used in this study is in Table 1, and a mosaic of the longest exposures for each field is shown as Figure 1. Details of UIT hardware, calibration, operations, and data reduction are in Stecher et al. 1992 and Stecher et al. 1996. Data discussed here are from images made with the $\lambda_{e f f}=1620 \AA$ filter, hereafter called 'B5'. Astro-1 data also include short exposures of Field 1 made in a near-UV $\left(\lambda_{e f f}=2490 \AA\right)$ and an additional far-UV $\left(\lambda_{e f f}=1520 \AA\right)$ bandpass (cf Cornett et al. 1994) which are not discussed here. (A discussion and analysis of UIT imaging of the Large Magellanic Cloud is given in Parker et al. 1996.)

UIT film images are reduced to linearized arrays and absolutely calibrated by comparison to other UV spectrophotometry as described in Stecher et al. 1992. The calibration for Astro-2 data has been revised using flight observations of standard fields (Stecher et al. 1996); estimated absolute uncertainty is $15 \%$ for well-exposed pixels.

An IDL/UIT implementation of DAOPHOT (Stetson 1987; J.K.Hill et al. 1993) is used to locate stars and perform aperture and PSF-fit photometry. Both aperture and PSF-fit photometry use a 2.9-arcsec (2.5-pixel) radius, which sets the limit for resolution of blended stars and the minimum size for non-stellar sources. PSF-fit photometry, using parameters chosen for images individually, is used for subsequent analysis of stellar objects. Astrometric solutions are derived for UIT images using HST guide stars as standards (Lasker et al. 1989). 11306 stars are measured, and positions, magnitudes, and errors for these objects are provided in the AAS CD-ROM series. The file includes UIT star number, field number, $\mathrm{x}$ and $\mathrm{y}$ positions in UIT pixels, right ascension and declination, $\mathrm{m}(162)$ derived from PSF photometry, and estimated error in $\mathrm{m}(162)$.

For the SMC data, IUE observations are used to measure combined aperture corrections and improved single-field absolute calibrations. The corrections are determined by integrating well-exposed and calibrated (Bohlin et al. 1990) IUE spectra of isolated field stars in each UIT image, and comparing the results with UIT PSF photometry. The number of IUE spectra used for each field ranges from 12 to 31. Statistical uncertainties in mean IUE/UIT ratios range from .07 to .02 mag, with larger uncertainties for longer UIT exposures because of the overexposure of the IUE sources on those images. The IUE/UIT ratio thus derived agrees with the nominal UIT absolute calibration within 10\%, after application of a directly measured aperture correction. UIT magnitudes are defined by $\mathrm{m}_{\lambda}=-2.5 \log \left(\mathrm{F}_{\lambda}\right)-21.1$, where $\mathrm{F}_{\lambda}$ is in $\operatorname{erg}\left(\mathrm{cm}^{2} \AA \mathrm{s}\right)^{-1}$.

Data from multiple exposures of each field are combined by selecting data from individual exposures which maximize the signal-to-noise ratio. For stellar images and pixels which are common to two fields, data are used from the field with the longer maximum exposure time, after signal-to-noise selection. In practice signal-to-noise selection is achieved 
for stellar photometry by restricting each exposure to a selected stellar magnitude range; this avoids systematic errors at the extremes of the UIT dynamic range on single exposures. For photometry of non-stellar objects, an extended-dynamic-range, calibrated composite image is produced for each field by 1 ) boxaveraging all exposures to a $\sim 4.5$ arcsec pixel size (to minimize the effects of possible densitometry misalignments of up to $\sim 1.12$ arcsec), and 2) selecting and using pixels from the individual boxaveraged frames with exposure values which maximize signal-to-noise ratios. This composite image is subsequently used for all extended source photometry.

\section{Far-UV Morphology}

Figure 11 shows that FUV emission from the SMC originates mostly in hot stellar populations which, while not restricted to clusters, are significantly clumped. No diffuse FUV emission is readily apparent (but see Section 5). The brightest features in the UIT images are the clusters NGC 346 and NGC 330, with additional FUV concentrations approximately centered in fields 1 and 2 . The bright FUV concentrations, spaced along the Bar centered at $\sim 0.5 \mathrm{kpc}$ intervals, have similar clustering and distribution properties to those evident in wide-field FUV images of the LMC (Smith, Cornett, and Hill 1987).

Comparison of large-scale $\mathrm{H} \alpha$ and FUV morphologies (Figure 2 a) and b)); Bothun, personal communication) can reveal spatial and temporal sequences of star formation over timescales of $\sim 20 \mathrm{Myr}$ (R.S.Hill et al. 1994). Ionized hydrogen emission marks the locations of the earliest-type stars, while detectible FUV flux is emitted by stars as late as A0. Therefore, $\mathrm{H} \alpha$ is strong for clusters up to a few million years of age, while detectible FUV emission continues for about 20 Myr. Figures 2a) and b) show that FUV concentrations cover a range of ages, including cases with coincident bright young $\mathrm{H} \alpha$ sources at the $\mathrm{NE}$ and SW ends of the bar, as well as evolved regions without $\mathrm{H} \alpha$.

UIT field 2, near the Bar's center, provides a intriguing instance of what appears to be sequential star formation. A ring of FUV-bright stars with a radius of about 4-5 arcmin ( 70-90 pc at a distance of $60 \mathrm{kpc}$; Hutchings 1982) dominates the field. Figures 2 a) and b) show that the stellar ring is bounded by, and is apparently interacting with, ionized hydrogen in a concentric, larger ring (radius $7-8$ arcmin or $\sim 120-140 p c$ ). Supernova remnant 0050-728 (Mathewson et al. 1984), outlined by the circle in Figure 2b, lies at the northern edge of the shell. HI observations (Staveley-Smith et al. 1995, Staveley-Smith et al. 1996) show loops comparable in size to the $\mathrm{H} \alpha$ ring near these locations at a range of velocities between about 90 and $130 \mathrm{~km} \mathrm{~s}^{-1}$; the ellipse in Figure ab shows the location of the ring seen at $\sim 110 \mathrm{~km} \mathrm{~s}^{-1}$. Fabry-Perot $\mathrm{H} \alpha$ measurements (Okumura 1993) 
show linewidths of up to several tens of $\mathrm{km} \mathrm{s}^{-1}$, reinforcing evidence for ties between the distribution of hot stars and the gas dynamics. In a simple model, the hollow-shell morphology of the HII has been driven by winds from now-defunct hot stars and supernova remnants, which have also propagated star formation from the center outward, as evidenced by relatively gas-free stars in the center of the HII ring. UIT-based detailed analysis of sequential star formation in this region will be the subject of future work Cornett et al. 1996). For example, comparing the distribution of UV-bright stars with that of $\mathrm{H} \alpha$ emission-line stars and small nebulae as catalogued by (Meyssonier \& Azzopardi 1993) will reveal any systematic stellar age gradients in the 0-30 Myr range.

\section{Stellar Photometry}

Luminosity functions for the four fields, normalized to the number of stars in each for $14.0<\mathrm{m}(162)<14.5$, are shown in Figure 3. From turnovers of the luminosity functions we estimate the limiting FUV magnitudes of the fields to be 14.5, 14.5, 14.5, and $15.0( \pm \sim 0.5)$ for fields 1-4 respectively, consistent with relative exposure times.

Luminosity functions for fields 1 and 2 show features that may be confirmed from other data. The peak for $11.0<\mathrm{m}(162)<11.5$ in field 1's luminosity function (solid line) is clearly displayed in the field's color-magnitude diagram as a supergiant "plume" of stars leaving the main sequence (Cornett et al. 1994). Field 2 (dashed line) shows a significant overabundance of stars with $10.0<\mathrm{m}(162)<11.0$ (more than $3 \sigma$ overabundant compared to each of the other three fields individually) which may be related to sequential star formation in that field. The high-luminosity ends of these $\mathrm{m}(162)$ distributions evidently show structure which reflects recent star formation.

As shown in previous UIT results (e.g. J.K.Hill et al. 1995) FUV sources are generally well-correlated with $\mathrm{H} \alpha$ emission. We have compared FUV point-source locations with those of the $711 \mathrm{H} \alpha$ emission-line stars and small nebulae within the UIT fields of view (Meyssonier \& Azzopardi 1993), finding 520 matches at a matching radius of 7.2 arcsec (approximately twice the estimated 1- $\sigma$ the UIT astrometric uncertainty for these images). As described above, a detailed comparison of the spatial distribution of FUV sources with and without associated $\mathrm{H} \alpha$ emission may provide insights into possible sequential star formation in the SMC.

We have also correlated our stellar photometry tables with the ground-based stellar photometry compiled by Azzopardi \& Vigneau (1982; hereafter AV) by positionally matching sources. The precision to which stellar positions are quoted in AV $(\sim 0.1$ arcmin $)$ 
does not permit unambiguous identification of stars fainter than, typically, m(162) 15 because several such stars typically occur in a part of a UIT image of that size. For AV supergiants, this $\mathrm{m}(162)$ limit corresponds to a spectral type of $\sim \mathrm{F} 0$; therefore, we do not identify or list $\mathrm{m}(162)$ values for AV stars of spectral type F0 or later. (Where available we have used the spectral types listed in AV from Azzopardi 1981 and other sources which employ medium resolution slit spectra as the basis for spectral classification.) Under this spectral type restriction we find 191 sources matched of the 195 within the UIT fields of view. Results of the photometry of these objects are presented in Table 2.

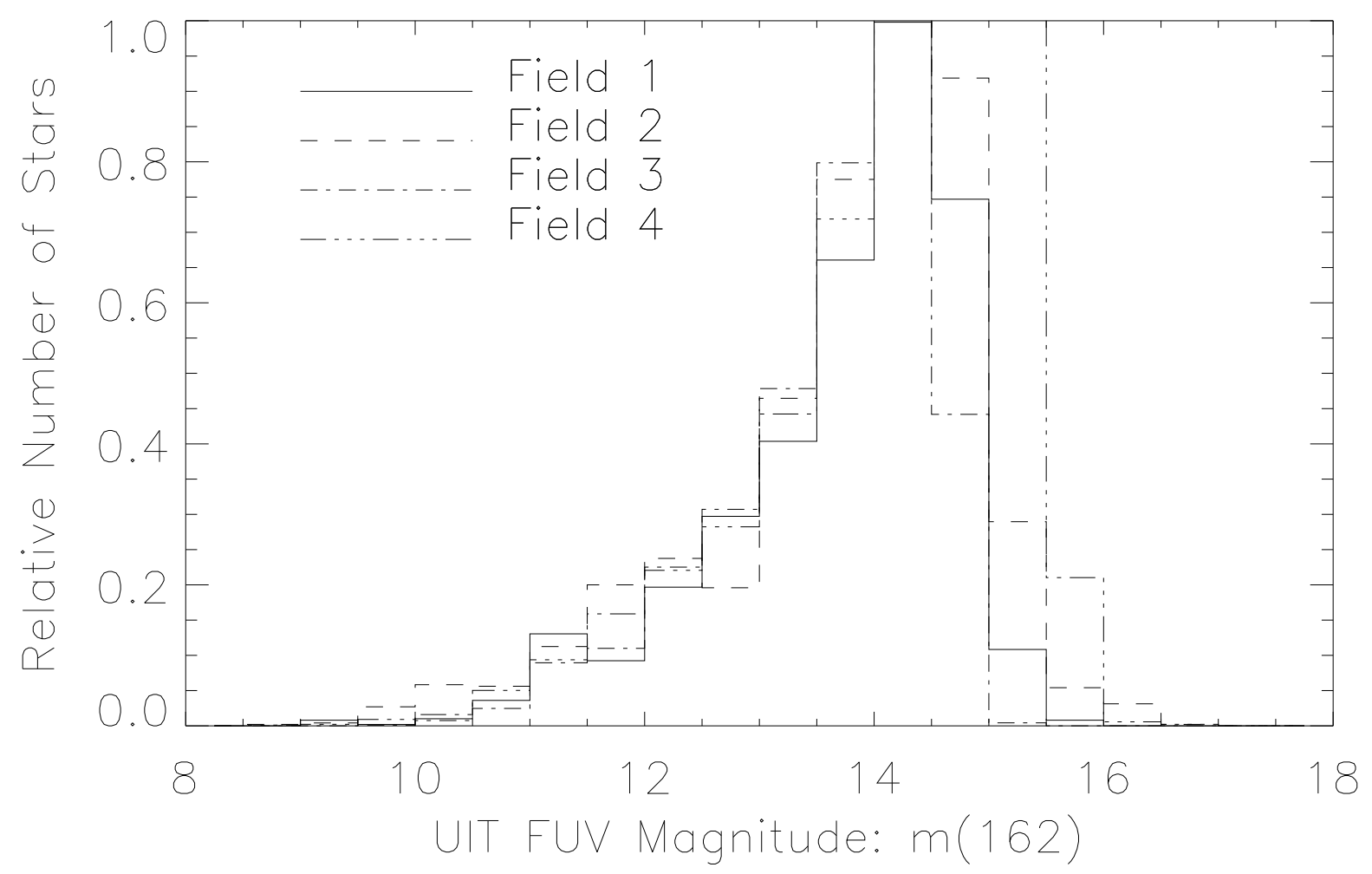

Fig. 3.- $\mathrm{m}(162)$ luminosity functions for the four fields, normalized to the number of stars in each for $14.0<\mathrm{m}(162)<14.5$. The luminosity functions for fields 1 and 2 show features that are confirmed by other data. The peak for $11.0<\mathrm{m}(162)<11.5$ in field 1's luminosity function (solid line) is clearly displayed in the field's color-magnitude diagram as a supergiant "plume" of stars leaving the main sequence (Cornett et al. 1994). In field 2 (dashed line), the overabundance of stars with $10.0<\mathrm{m}(162)<11.0$ may be attributable to the effects of sequential star formation in the field. 


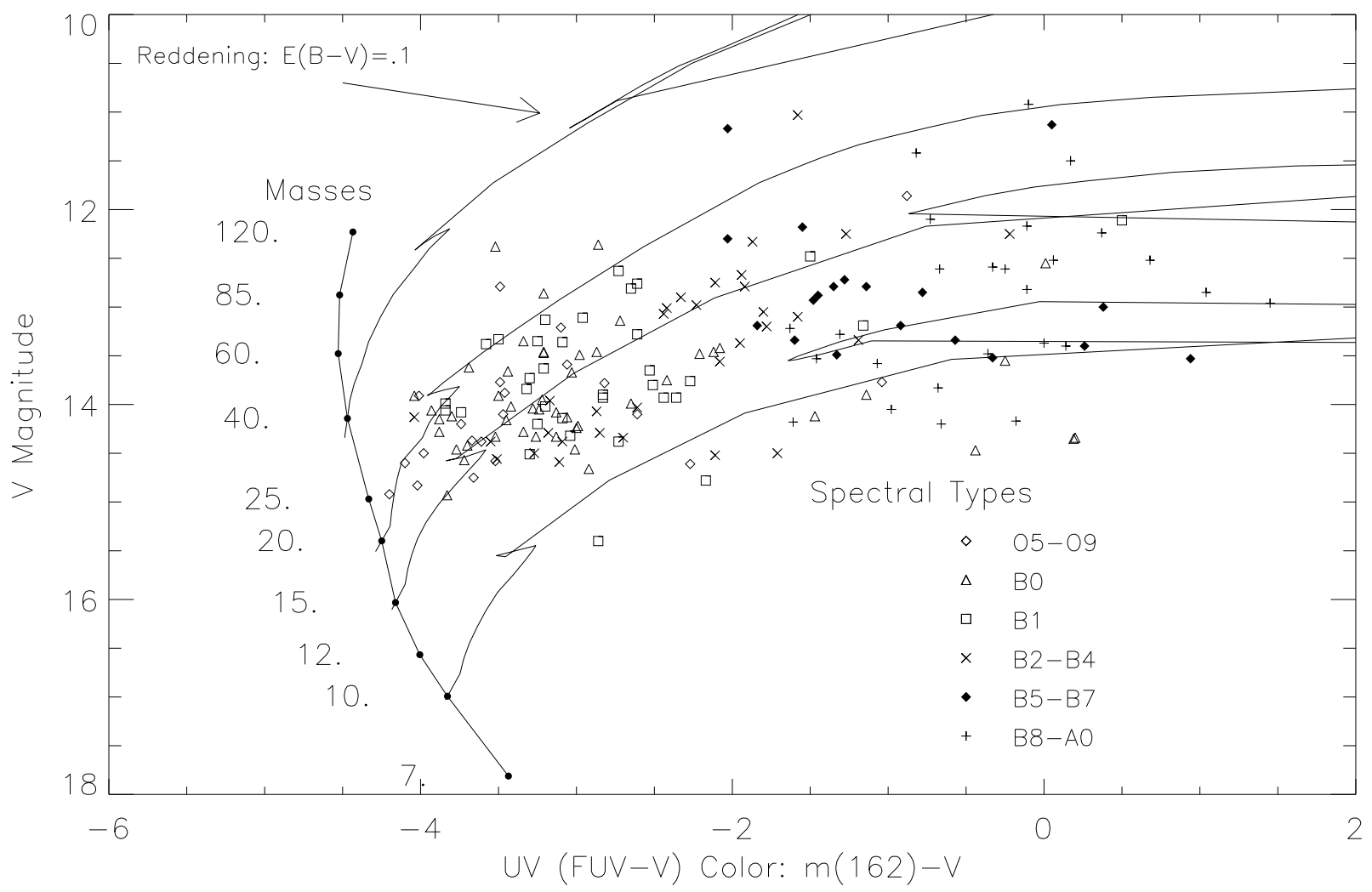

Fig. 4.- The $(\mathrm{m}(162)-\mathrm{V}), \mathrm{V}$ color-magnitude diagram for 191 stars in common with the catalog of Azzopardi \& Vigneau (1982). Discrete symbols are observed stars, with spectral types as noted in the figure. No reddening corrections have been applied to the observed data. The solid lines show SMC-composition stellar models produced by using model atmospheres of Kurucz 1992 on stellar evolution models of Charbonnel et al. 1993. Filled circles outline a 1 Myr isochrone, with masses marked; the tracks to the upper right show the subsequent evolutionary paths of $10,15,20$, and $40 \mathrm{M}_{\odot}$ stars. Model stars are adjusted to distance modulus 18.9 and foreground Galactic extinction $\mathrm{E}(\mathrm{B}-\mathrm{V})=0.02$ magnitudes (Hutchings 1982). The reddening vector shown, using the SMC extinction law of Hutchings 1982 and $\mathrm{E}(\mathrm{B}-\mathrm{V})=0.1$, is a typical large value for the SMC (Westerlund 1990). The evolutionary tracks show that these stars predominantly have masses between 10 and $20 \mathrm{M}_{\odot}$ and imply ages between 10 and $30 \mathrm{Myr}$. The lower limit corresponds to a real absence of supergiant stars brighter than about $\mathrm{V}=11$. The general segregation of spectral types by color implies that much of the $(\mathrm{m}(162)-\mathrm{V})$ color variation seen in this figure is due to the intrinsic colors of the stars themselves. 
Figure 4 is a color-magnitude diagram of the 191 identified stars common to our data and AV, with spectral classes differentiated by different symbols. No reddening corrections have been applied to observed data. SMC-composition stellar models are produced by using model atmospheres of Kurucz 1992 with $\log \left(\mathrm{Z} / \mathrm{Z}_{\odot}\right)=-0.5(\sim$ solar/3.2), on stellar evolution models of Charbonnel et al. 1993 with $\mathrm{z}=0.004$ ( $\sim$ solar $/ 5$.). These grid points are selected as models with composition parameters nearest to the SMC's ( solar/4; Westerlund 1990). Filled circles outline a 1 Myr isochrone, with masses marked; the tracks to the upper right show the subsequent evolutionary paths of 10, 15, 20, and $40 \mathrm{M}_{\odot}$ stars. Model stars are adjusted to distance modulus 18.9 and foreground Galactic extinction $\mathrm{E}(\mathrm{B}-\mathrm{V})=0.02$ magnitudes (Hutchings 1982). The reddening vector shown, using the SMC extinction curve of Hutchings 1982 and $\mathrm{E}(\mathrm{B}-\mathrm{V})=0.1$, is a typical large value for the SMC (Westerlund 1990). The large FUV/V leverage of extinction in the $\mathrm{SMC}\left(\mathrm{A}_{162} / \mathrm{E}(\mathrm{B}-\mathrm{V})=15.75\right)$ is reflected in the nearly-horizontal orientation of the reddening vector.

The stars catalogued by AV, the visually brightest non-cluster stars in the SMC, are known to be supergiants of a range of spectral types. The evolutionary tracks in Figure 4 clearly demonstrate that these stars predominantly have masses between 10 and $20 \mathrm{M}_{\odot}$, and therefore, ages between 10 and $30 \mathrm{Myr}$. The upper age limit is set by the faintness limit of the AV data; however, the lower limit corresponds to a real absence of supergiant stars brighter than about $\mathrm{V}=11\left(\mathrm{M} \sim 30 \mathrm{M}_{\odot}\right.$.) While the effects of some reddening (seen as excesses of $(\mathrm{m}(162)-\mathrm{V})$ color of up to 1.5) are apparent, most color variation seen in this figure is due to the intrinsic color variations of the stars themselves, as seen from the general segregation of spectral types by color.

Figure 5 illustrates the strong variation in (FUV-V) color with spectral type. The data points are the observed colors, as in Figure 4, plotted against spectral type from AV. The solid line is a spline fit to the observed $(\mathrm{m}(162)-\mathrm{V})$ colors of Galactic supergiants from the atlas of Fanelli et al. 1992. The atlas colors have been reddened by the amount of the SMC's Galactic foreground reddening. The sensitivity of the $(\mathrm{m}(162)-\mathrm{V})$ color to spectral type is evident, as are the significant reddenings for some stars, caused by the large FUV leverage of the SMC extinction law. The atlas colors are seen to form a blue limit for the observed colors, as expected for unreddened stars.

While a true calibration of color versus spectral type for SMC stars is not available, Galactic values are probably appropriate for blue stars: $(\mathrm{m}(162)-\mathrm{V})$ colors of Galacticand SMC-composition model atmospheres differ by less than 0.10 for models that have $(\mathrm{m}(162)-\mathrm{V})<-1.0$. However, at Galactic $(\mathrm{m}(162)-\mathrm{V})=1.5$, SMC models are approximately $0.5 \mathrm{mag}$ bluer, with larger differences for redder stars. Therefore, much of the apparent discrepancy between the blue limit of the observed colors and the atlas 
colors is probably due to the fact that the locus of colors from an "SMC atlas" would be significantly bluer for stars later than about A0, than the Galactic atlas colors shown.

Figure 5 provides a straightforward method of computing the reddenings along the lines of sight to the stars in AV. We do so by comparing the observed colors to atlas colors for the spectral type, corrected for Galactic foreground reddening, and converting the difference to $\mathrm{E}(\mathrm{B}-\mathrm{V})$ using the extinction law of Hutchings 1982. Extinctions computed in this way are presented in Table 2 for all identified AV stars. Stars later in spectral type

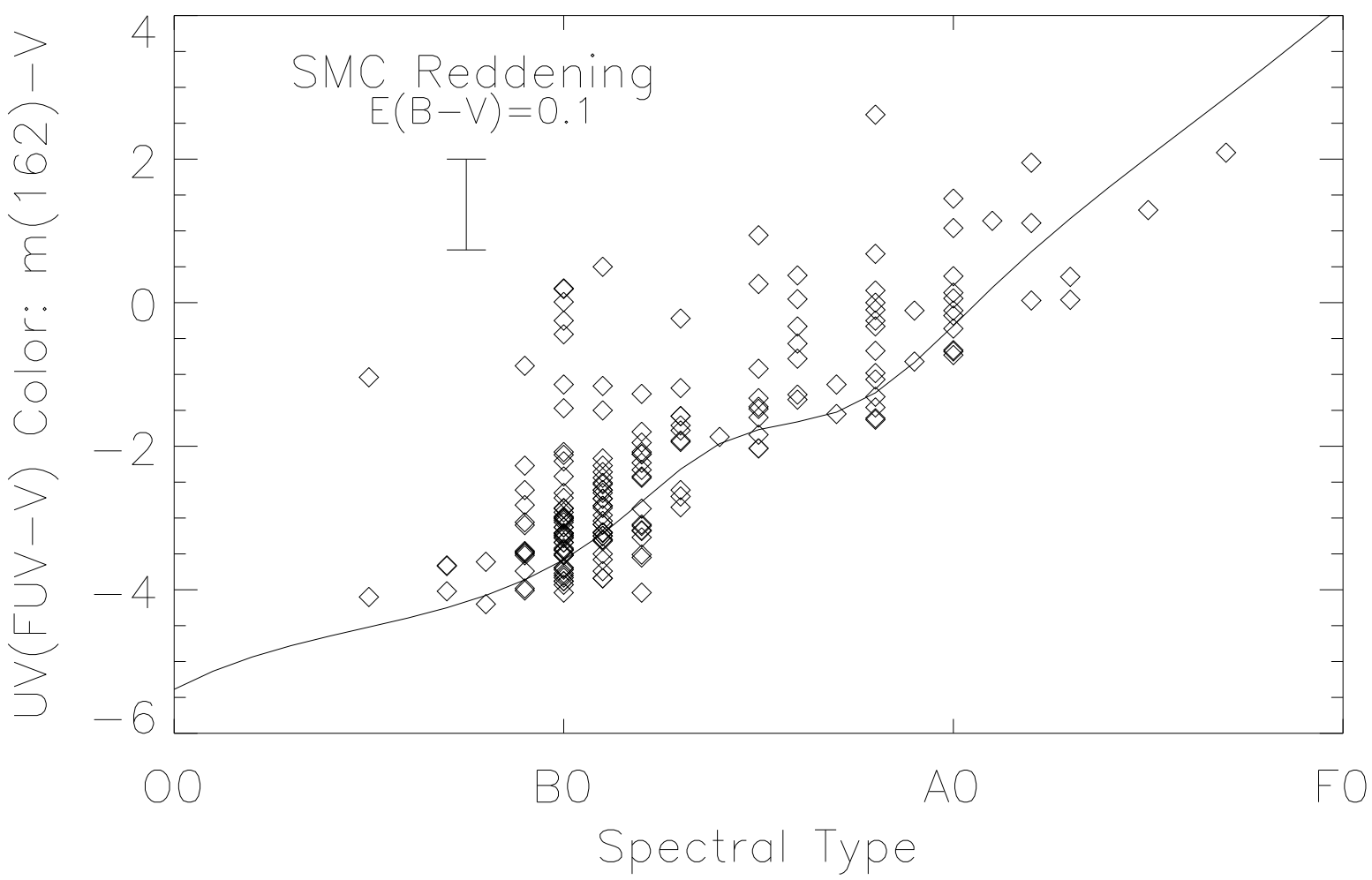

Fig. 5.- Diamonds: Stellar spectral type vs $(\mathrm{m}(162)-\mathrm{V})$ color for stars from the catalog of Azzopardi \& Vigneau (1982). Where available, spectral types listed in Azzopardi \& Vigneau from Azzopardi 1981 and other sources are used, which employ medium resolution slit spectra as the basis for spectral classification. No reddening corrections have been applied to the observed color data. Solid line: Spline fit to the $(\mathrm{m}(162)-\mathrm{V})$ colors derived from the spectral atlas of unreddened Galactic stars of Fanelli et al. 1992, reddened by $\mathrm{E}(\mathrm{B}-\mathrm{V})=0.02$. Galactic and SMC stellar $(\mathrm{m}(162)-\mathrm{V})$ colors differ by less than 0.10 for stars bluer than $(\mathrm{m}(162)-\mathrm{V})=-1.0 ;$ SMC stars are significantly bluer than Galactic stars beyond that limit. 
than $\mathrm{B} 8$, which have uncertain intrinsic colors, are noted.

Figure 6 shows the resulting $\mathrm{E}(\mathrm{B}-\mathrm{V})$ values plotted at the locations of the stars on the $\mathrm{SMC}$, against the $\mathrm{H} \alpha$ image shown in Figure 2a. All values of $\mathrm{E}(\mathrm{B}-\mathrm{V})>\sim 0.15$ may be

seen from Figure $2 \mathrm{~b}$ to be at least along the line of sight to $\mathrm{H} \alpha$ emission. The correlation of large reddening values with location implies that these stars are probably associated with the nebulosity.

In the future UIT photometry of the SMC will be supplemented by additional ground-based observations currently underway by the UIT team, as well as by the comprehensive photometric surveys of the Magellanic Clouds (Zaritsky 1996).

\section{HII Region Photometry}

$\mathrm{H} \alpha$ emission from HII regions indirectly traces the OB star content of the regions via the process of nebular ionization by the hot stars and subsequent recombination of

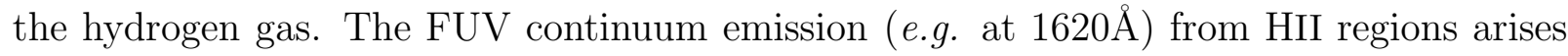
from $\mathrm{OB}$ stars as well as from the more numerous non-ionizing stars (mid-B through early A types.) Therefore, the comparison of $\mathrm{H} \alpha$ and FUV fluxes from HII regions provides a sensitive measure of each HII region's evolutionary state. Evolutionary models of single-burst ionizing clusters confirm that the ratio of Lyman continuum to FUV flux rapidly decreases with age, with the FUV emission remaining significant for $\sim 20 \mathrm{Myr}$ (R.S.Hill et al. 1994).

We have measured FUV fluxes for $42 \mathrm{H} \alpha$-bright HII regions from the catalog of Davies, Elliott, and Meaburn 1976 (hereafter DEM) that were measured at $\mathrm{H} \alpha$ by Kennicutt \& Hodge 1986 (hereafter [KH), and have compared the observed flux ratios with predictions from cluster models. We compute FUV fluxes in two ways: by summing the flux from stars in the apertures defined by $\mathrm{KH}$; and by summing all flux in pixels in the apertures- the latter method including diffuse FUV emission. (Our use of the KH aperture definitions causes significant aperture overlaps in a few cases.) Table 3 presents fluxes and errors: $\mathrm{H} \alpha$ from KH; FUV from stars (from PSF fits to stars in the aperture); and FUV total fluxes from the apertures themselves.

For the FUV aperture measurements the "sky" flux has been set to zero, and the error term stated does not include a sky contribution. We choose this approximation because the actual sky contribution to any FUV aperture measurement or its uncertainty is very small, and any aperture inside the UIT image area will measure a high background due to SMC material. We set limits on true sky flux by measuring the average background in Astro-2 
images made in the UIT B5 filter of a nearby field containing part of the globular cluster 47 Tuc. These images, long exposures (1030 and $1800 \mathrm{sec}$ ) made during orbital day, have average earth limb elevation angles and other observational conditions which are typical of those during UIT SMC observations, and therefore give representative background measurements (Waller et al. 1995). As measured in this way the sky contribution to the FUV aperture fluxes is $3.3 \times 10^{-18} \mathrm{erg}\left(\mathrm{cm}^{2} \AA \mathrm{s} \text { arcsec }{ }^{2}\right)^{-1}$, with an average contribution of $0.75 \%$ and a maximum of $1.60 \%$ to the individual aperture fluxes. These estimates of "sky" contributions are also listed in Table 3.

Figure 7 shows the data from Table 3. Open diamonds are aperture fluxes, and crosses are stellar photometry. No corrections for Galactic foreground reddening have been made to the data. The FUV flux is generally well correlated with its $\mathrm{H} \alpha$ counterpart; furthermore, the ratio of aperture to stellar FUV flux is relatively uniform, especially for brighter HII regions. This ratio measures the relative amounts of bright-star and "diffuse" light, where "diffuse" light here includes contributions from faint stars as well as from dust scattering. The mean value of the ratio of aperture flux to stellar flux, averaged in the log ratio for all HII regions, is 1.79; weighted by flux (the total aperture flux for all HII regions divided by the total stellar flux for all HII regions) is 2.15. Two lines of argument point to a dust-scattering origin for most of the additional FUV flux, however. First, Bohlin et al. 1982 have determined the total-to-stellar flux ratio for the Orion nebula, for which the number of undetected stars is small, to be 2.5 for $200-300 \AA$ bandpasses near 1400,1823 , 2242 , and $2622 \AA$. Second, extrapolating our measured luminosity function for the Field 4, which has the deepest exposure, to $\mathrm{m}(162)=18.0$ (corresponding to an A0 main sequence star, at which spectral type the FUV flux drops rapidly) predicts an additional stellar flux contribution of $22 \%$ and a resulting flux-weighted total-to-stellar ratio of 1.76- significantly smaller than the observed value. Scattered-light fractions near the observed SMC value are found in the giant HII regions of M33 (Malumuth et al. 1996). Although the small SMC dust abundance (Westerlund 1990) undoubtedly causes the scattering to be significantly less than for Orion, it is nonetheless clear that dust-scattered radiation is a major contributor to the FUV aperture emission from HII regions.

Figure 7 also displays the $\mathrm{FUV} / \mathrm{H} \alpha$ ratio, known to be a good diagnostic of HII region evolution (R.S.Hill et al. 1994). We have computed $\mathrm{F}(162) / \mathrm{H} \alpha$ ratio values for clusters with a single burst of star formation of SMC composition using the IDL procedure CLUSTFLUX (Landsman, personal communication). The ratio rises essentially monotonically from 0.1 at 1 Myr to 4.3 at $10 \mathrm{Myr}$; ratios for cluster ages of $1 \mathrm{Myr}$, 3Myr, 6.5 Myr, and 8.5 Myr, corrected for foreground Galactic reddening (which increases all $\mathrm{F}(162) / \mathrm{H} \alpha$ values by 10\%) are plotted as solid lines in Figure 7. For reference, the arrow in Figure 7 shows a typical internal SMC extinction of $\mathrm{E}(\mathrm{B}-\mathrm{V})=0.1$; Caplan et al. 1996 find reddenings local 
to $\mathrm{SMC}$ bar HII regions in the range $0.08<\mathrm{E}(\mathrm{B}-\mathrm{V})<0.27$ by comparing 6 -cm radio, $\mathrm{H} \alpha$, $\mathrm{H} \beta$, and nearby stellar measurements. Therefore, model and observed ratios agree well for HII-region ages of a few Myr and reasonable internal SMC extinction; the observed HII regions are evidently no older than $~ 5 \mathrm{Myr}$ for any internal SMC reddening, and, for typical assumed SMC reddening, are between $1 \mathrm{Myr}$ and $3 \mathrm{Myr}$ in age. This result is consistent with observations of HII regions in galaxies as disparate as NGC 4449 R.S.Hill

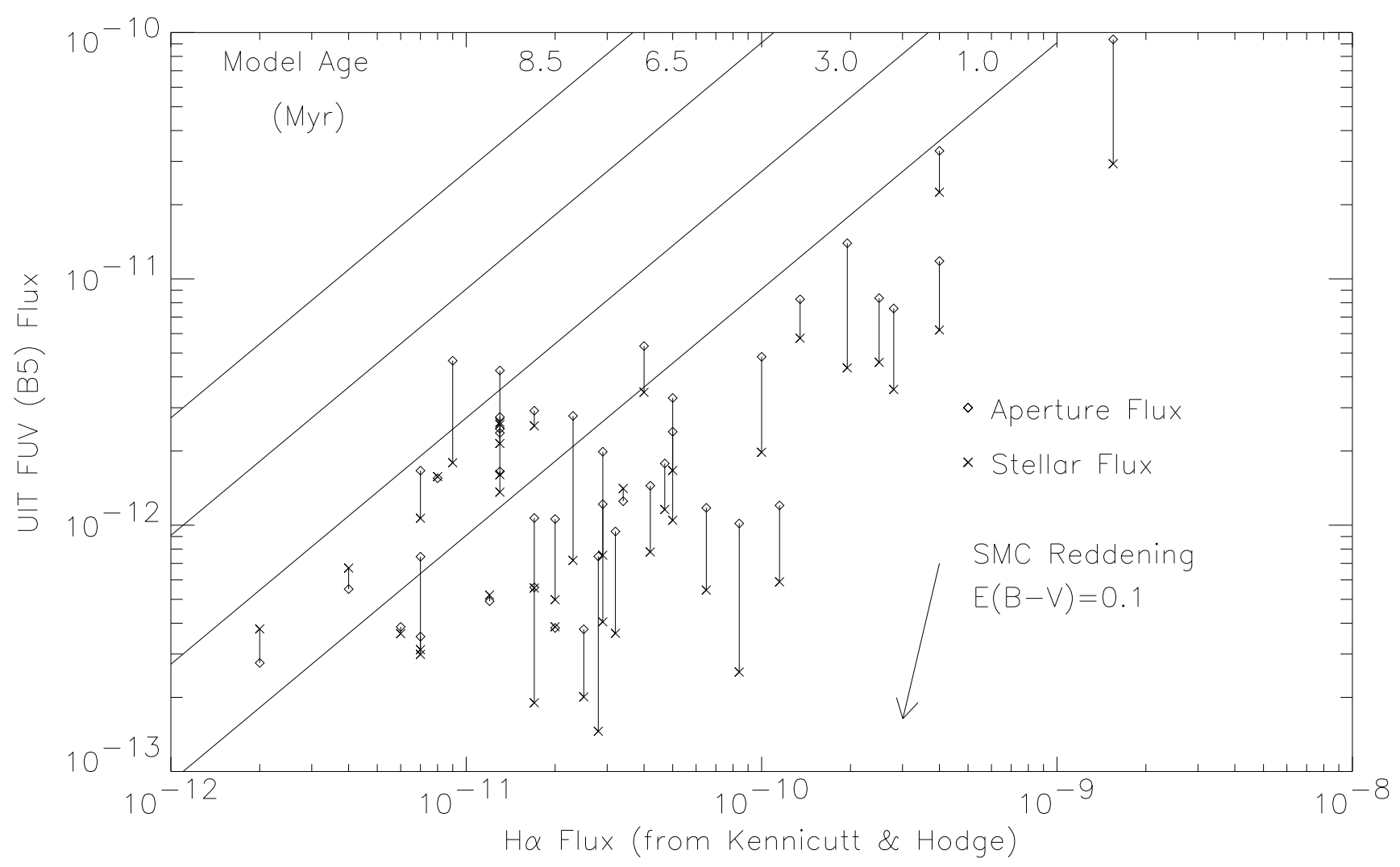

Fig. 7.- FUV $\left(\lambda_{e f f}=1620 \AA\right)$ vs $\mathrm{H} \alpha$ flux for HII regions as defined by the apertures of Kennicutt \& Hodge (1986). Crosses mark the total FUV stellar flux, from our stellar photometry, of stars which lie within the KH apertures. Diamonds mark the total FUV flux contained within the apertures. No reddening corrections are applied to the data, although a typical SMC internal reddening corresponding to $\mathrm{E}(\mathrm{B}-\mathrm{V})=0.1$ is shown. Solid lines at constant $\mathrm{FJV} / \mathrm{H} \alpha$ mark the expected $\mathrm{FUV} / \mathrm{H} \alpha$ ratios for clusters of single-burst formation of stars of SMC composition, reddened to Galactic foreground values. The ages of the clusters are $1 \mathrm{Myr}, 3 \mathrm{Myr}, 6.5 \mathrm{Myr}$, and 8.5 Myr respectively, with the smallest FUV/H $\alpha$ value corresponding to age 1 Myr. 
et al. 1994) and M81 (J.K.Hill et al. 1995). There is also a clear tendency for H $\alpha$-bright HII regions to be either relatively young- which is not surprising- or unreddened. The absence of significantly older HII regions is most likely a selection effect originating in KH's bias for $\mathrm{H} \alpha$-bright HII regions.

\section{Summary}

UIT FUV images and derived stellar photometry for most of the Bar of the SMC are presented. The UV morphology of the SMC's Bar shows four concentrations of UV-bright stars spread from northeast to southwest at nearly equal ( $\sim 30$ arcmin) spacings. One of the concentrations, near DEM 55, appears as a well-defined 8-arcmin diameter ring surrounded by a larger $\mathrm{H} \alpha$ ring and strongly suggests sequential star formation.

FUV PSF photometry, resulting in m(162) magnitudes, is obtained for 11,306 stars. We present a FUV luminosity function for the SMC bar, complete to $\mathrm{m}(162) \sim 14.5$, and compare our photometry with the compiled ground-based data of AV. Detected objects are well correlated with other SMC Population I material; 520 of $711 \mathrm{H} \alpha$ emission-line stars and small nebulae within the UIT fields of view are identified with FUV sources. For early type stars, the bluest observed $(\mathrm{m}(162)-\mathrm{V})$ colors for each spectral type agree well with values computed from unreddened Galactic spectral atlas stars for types earlier than about A0; for later types, observed SMC stars range significantly bluer, as predicted by low-metallicity models. We attribute redder colors for some stars of all spectral types to strong FUV extinction due to even small amounts of SMC dust. Internal SMC reddenings are determined for all catalog stars. All stars with $\mathrm{E}(\mathrm{B}-\mathrm{V})>0.15$ are within regions of visible $\mathrm{H} \alpha$ emission.

FUV photometry is obtained for the resolved stars and for the total emission from DEM HII regions in the SMC Bar. The flux-weighted ratio of total to stellar flux for DEM HII regions using the apertures of $\mathrm{KH}$ is 2.15 ; since only $22 \%$ more flux is contributed by stars fainter than $\mathrm{m}(162)=14.5$, most of the excess total flux is due to scattered FUV from dust. Stellar and total emission from DEM HII regions are well correlated with $\mathrm{H} \alpha$ fluxes measured by KH. We compute ratios of FUV to $\mathrm{H} \alpha$ flux for 42 SMC HII regions and compare them with model results, finding that the observed ratios for all $\mathrm{H} \alpha$-selected HII regions are consistent with models of SMC metallicity, ages from 1-5 Myr, and moderate $(\mathrm{E}(\mathrm{B}-\mathrm{V})=0.0-0.1 \mathrm{mag})$ extinction.

We gratefully acknowledge the contributions made by the many people involved in the Astro-2 mission. We thank Robert S. Hill, Wayne Landsman, and Michael Fanelli for useful 
discussions, Greg Bothun for the use of his $\mathrm{H} \alpha$ image of the SMC, Joel Offenberg for help in preparing publication-quality images, and Joan Hollis and Emily Zamkoff for data entry and programming assistance. We also thank Lister Staveley-Smith for kindly supplying a digital version of a part of his HI maps of the complete SMC.

Funding for the UIT project has been through the Spacelab Office at NASA under

project number 440-51. RWO gratefully acknowledges NASA support of portions of this research through grants NAG5-700 and NAGW-2596 to the University of Virginia.

\section{REFERENCES}

Azzopardi, M. 1981, Ph.D. Thesis, Paul Sabatier University of Toulose, No. 979.

Azzopardi, M. \& Vigneau, J. 1982, A\&AS 50, 291.

Bohlin, R.C., Hill, J.K., Stecher, T.P., and Witt, A.N. 1982, ApJ 255, 87.

Bothun, G. (personal communication).

Caplan, J., Ye, T., Deharveng, L., Turtle, A., and Kennicutt, R. 1996, A\&A 307, 403.

Charbonnel, C., Meynet, G., Maeder, A., Schaller, G., \& Schaerer, D. 1993, A\&AS 101, 415.

Cornett, R.H., Hill, J.K., Bohlin, R.C., O’Connell, R.W., Roberts, M.S., Smith, A.M., \& Stecher, T.P. 1994, ApJ 425, L117.

Cornett et al. 1996, in preparation.

Davies, R.D., Elliott, K.H., and Meaburn, J. 1976, MmRAS 81, 89.

Fanelli, M.N., O’Connell, R.W., Burstein, D. \& Wu, C.-C. 1992, ApJS 82, 197.

Hill, J.K., Bohlin, R.C., Cheng, K-P., Fanelli, M.N., Hintzen, P.M.N., O’Connell,R.W., Roberts, M.S., Smith, A.M., Smith, E.P., \& Stecher, T.P. 1993, ApJ 413, 610.

Hill, J.K., Cheng, K-P., Bohlin, R.C., Cornett, R.H., Hintzen, P.M.N., O’Connell,R.W., Roberts, M.S., Smith, A.M., Smith, E.P., \& Stecher, T.P. 1995, ApJ 438, 182.

Hill, R.S., Home, A.T., Smith, A.M., Bruhweiler, F.C., Cheng, K.-P., Hintzen, P.M., and Oliverson, R.J. 1994, ApJ 430,568.

Hutchings, J.B. 1982, ApJ 255, 70. 
Kennicutt, R.C. Jr. \& Hodge, P.W. 1986, ApJ 306,130.

Kurucz, R.L. 1992, in The Stellar Populations of Galaxies, ed. B. Barbuy \& A. Renzini (Dordrecht: Kluwer Academic), 225.

Landsman, W.B. (personal communication).

Lasker, B.M., Sturch, C.R., McLean, B.J., Russell, J.L., Jenkner, H. \& Shara, M.M. 1989, Space Telescope Science Institute Preprint No. 363.

Malumuth, E.M., Waller, W.H., and Parker, J. Wm. 1996, AJ 111, 1128.

Mathewson, D.S., Ford, V.L., Dopita, M.A., Tuohy, I.R., Mills, B.Y., \& Turtle, A.J. 1984, ApJS 55, 189.

Meyssonier, N. and Azzopardi, M. 1993, A\&AS 102, 451.

Okumura, K., 1993, Ph.D. dissertation, University of Paris.

Parker, J. Wm. 1996, in preparation.

Smith, A.M., Cornett, R.H., and Hill, R.S. 1987, ApJ 320, 609.

Staveley-Smith, L., Sault, R.J., McConnell, D., Kesteven, M.J., Hatzidimitriou, D., Freeman, K.C., and Dopita, M.A. 1995, Pub. Astr. Soc. Austr. 12, 13.

Staveley-Smith, L., Sault, R.J., Hatzidimitriou, D., Kesteven, M.J., \& McConnell, D. 1996, MNRAS in press.

Stecher et al. 1992, ApJ 395, L1.

Stecher et al. 1996, submitted to PASP.

Stetson, P.B. 1987, PASP 99, 101.

Waller, W.H., Marsh, M., Bohlin, R.C., Cornett, R.H., Dixon, W.V., Isensee, J.E., Murthy, J., O’Connell,R.W., Roberts, M.S., Smith, A.M., \& Stecher, T.P. 1995 AJ 110, 1255.

Westerlund, B.E. 1990 A\&A Rev. 2,29.

Zaritsky, D.L. 1996, in preparation. 
Fig. 1.- A far-UV (FUV) mosaic of the Small Magellanic Cloud. It is made up of the longest FUV $\left(\lambda_{e f f}=1620 \AA\right)$ exposures of SMC fields made by UIT during the Astro-1 and Astro2 missions. Exposure times range from 117 to 898 seconds; see Table 1 for observational details. The bright clusters centered in Fields 4 and 3 respectively are NGC 346 and NGC 330 ; the field of view shown encompasses nearly the entire SMC bar, and the resolution is $\sim 3^{\prime \prime}$.

Fig. 2.- a) The SMC H $\alpha$ "parking lot camera" image obtained by Bothun (personal communication) displayed on a linear greyscale. - b) Linearly spaced contours from Figure 2a) superimposed on the image of Figure 1. A constant background has been subtracted. Note the prominent ring of $\mathrm{H} \alpha$ emission surrounding, and larger than, the ring of FUV-bright stars in Field 2. SNR 0050-728 (Mathewson et al. 1984) is marked in size and position by the circle at the northern edge of the ring. A prominent ring of HI emission, seen over a range of radial velocities centered at $\sim 110 \mathrm{~km} \mathrm{~s}^{-1}$ in the data of Staveley-Smith et al. 1995, is outlined by the ellipse.

Fig. 6. - Stellar reddening $(\mathrm{E}(\mathrm{B}-\mathrm{V}))$ map, computed by comparing the observed $(\mathrm{m}(162)-\mathrm{V})$ colors for stars from the catalog of Azzopardi \& Vigneau (1982) with the Galactic atlas spline fit for that spectral type shown in Figure 5. Stellar symbol diameter is proportional to $\mathrm{E}(\mathrm{B}-\mathrm{V})$ as shown in the legend. Observed colors have been corrected for Galactic foreground reddening. All values of $\mathrm{E}(\mathrm{B}-\mathrm{V})>\sim 0.15$ may be seen from Figure $2 \mathrm{~b}$ to be at least along the line of sight to $\mathrm{H} \alpha$ emission. The correlation of large reddening values with location implies that these stars are probably associated with the nebulosity. 
TABLE 1. Observational parameters for UIT images of the Small Magellanic Cloud.

TABLE 2. Observational data for stars from Azzopardi \& Vigneau 1982 (AV) which were observed by UIT. AV stars which are within UIT fields but later in spectral type than A9 are omitted, since they are too faint in the FUV for detection by UIT. m(162)values are derived from PSF photometry as described in the text. $\mathrm{E}(\mathrm{B}-\mathrm{V})$ values are derived by comparing the unreddened $(\mathrm{m}(162)-\mathrm{V})$ color for the stellar spectral type with the observed color, using the reddening law of Hutchings 1982.

TABLE 3. Fluxes for HII regions from the list of Davies, Elliott, \& Meaburn 1976 (DEM) as measured by Kennicutt \& Hodge 1986 (KH) which are completely contained within the UIT field of view. "FUV Stellar" flux is the total flux of stars within the aperture defined by KH; "FUV Aperture" flux is the total pixel flux contained within the aperture defined by KH; and "Sky Flux" is an estimate for the non-SMC sky contribution within the aperture, as described in the text. 
Table 1. UIT FUV Images of the SMC

\begin{tabular}{|c|c|c|c|c|c|c|}
\hline Field & $\begin{array}{c}\text { Field Center } \\
\text { RA (2000.0) Dec }\end{array}$ & $\begin{array}{c}\text { Exposure Times } \\
(\mathrm{sec})\end{array}$ & \multicolumn{2}{|c|}{$\begin{array}{l}\text { Obs. Epoch } \\
\text { (GMT) }\end{array}$} & $\begin{array}{l}\text { No. } \\
\text { Stars }\end{array}$ & Flight \\
\hline 1 & $004733.7-730626.8$ & $23.9,117.0$ & $12 / 05 / 90$ & $06: 16$ & 1713 & Astro-1 \\
\hline 2 & $005058.3-724456.5$ & $25.9,247.0$ & $03 / 16 / 95$ & $23: 50$ & 2110 & Astro-2 \\
\hline 3 & $005641.2-722828.8$ & $18.7,459.0$ & $03 / 06 / 95$ & $06: 14$ & 2517 & Astro-2 \\
\hline 4 & $005924.2-721108.4$ & $36.2,179.0,898$ & $03 / 09 / 95$ & 04:04 & 4966 & Astro-2 \\
\hline
\end{tabular}


Table 2. UIT FUV Magnitudes of AV Stars

\begin{tabular}{|c|c|c|c|c|c|c|c|c|}
\hline \multirow[t]{2}{*}{$\#$} & \multirow{2}{*}{$\begin{array}{c}\mathrm{AV} \\
\#\end{array}$} & \multirow{2}{*}{$\begin{array}{l}\mathrm{UIT} \\
\#\end{array}$} & \multirow{2}{*}{$\begin{array}{l}\text { R.A. }(2000.0) \text { Dec } \\
\text { (Azzopardi and }\end{array}$} & \multirow{2}{*}{$\begin{array}{l}\text { Sp } \\
\text { Jigneau) }\end{array}$} & \multirow{2}{*}{$\mathrm{V}$} & \multicolumn{2}{|c|}{ UIT } & \multirow[t]{2}{*}{$\mathrm{E}(\mathrm{B}-\mathrm{V})$} \\
\hline & & & & & & $\mathrm{m}(162)$ & $\sigma$ & \\
\hline 1 & $2 \mathrm{~B}$ & 1686 & $004351.2-730854$ & B0 & 13.49 & 10.51 & 0.10 & 0.05 \\
\hline 2 & 3 & 70 & $004429.6-725800$ & B0 & 14.12 & 12.65 & 0.07 & 0.17 \\
\hline 3 & 6 & 1699 & $004519.8-731518$ & B0 & 13.46 & 10.25 & 0.08 & 0.03 \\
\hline 4 & 7 & 1417 & $004533.7-730431$ & B0 & 14.57 & 10.85 & 0.06 & -0.01 \\
\hline 5 & 8 & 1505 & $004536.2-725912$ & B8 & 13.53 & 12.07 & 0.05 & -0.02 \\
\hline 6 & 9 & 1694 & $004537.1-731407$ & $\mathrm{~B} 2$ & 13.05 & 11.25 & 0.07 & 0.08 \\
\hline 7 & 11 & 1622 & $004554.3-731613$ & $\mathrm{~B} 2$ & 13.56 & 11.48 & 0.05 & 0.05 \\
\hline 8 & 12 & 1429 & $00462.8-730619$ & O9 & 13.21 & 10.11 & 0.07 & 0.06 \\
\hline 9 & 14 & 321 & $004633.2-730555$ & $\mathrm{O} 5$ & 13.77 & 12.73 & 0.07 & 0.28 \\
\hline 10 & 16 & 1558 & $004655.8-730825$ & B1 & 13.19 & 12.03 & 0.10 & 0.16 \\
\hline 11 & 17 & 1522 & $00473.6-730555$ & B1 & 13.76 & 11.49 & 0.12 & 0.08 \\
\hline 12 & $17 \mathrm{~A}$ & 1679 & $00474.8-730619$ & $\mathrm{~B} 2$ & 13.37 & 11.42 & 0.10 & 0.07 \\
\hline 13 & 18 & 1433 & $004713.1-730625$ & B1 & 12.48 & 10.98 & 0.04 & 0.14 \\
\hline 14 & 19 & 2766 & $004715.8-724955$ & B6 & 13.00 & 13.38 & 0.08 & 0.16 \\
\hline 15 & 20 & 158 & $004729.9-730125$ & B8: & 12.14 & 14.76 & 0.08 & 0.31 \\
\hline 16 & 21 & 1690 & $004732.6-731056$ & B1 & 14.14 & 11.05 & 0.05 & 0.01 \\
\hline 17 & 22 & 1444 & $004739.8-730738$ & B2 & 12.25 & 10.98 & 0.06 & 0.12 \\
\hline 18 & 23 & 1670 & $004740.1-732243$ & B3 & 12.25 & 12.03 & 0.08 & 0.17 \\
\hline 19 & 24 & 1675 & $004742.9-730225$ & O9 & 13.78 & 10.96 & 0.07 & 0.08 \\
\hline 20 & 25 & 1597 & $004747.5-731219$ & B5 & 13.19 & 11.35 & 0.10 & -0.01 \\
\hline 21 & 26 & 514 & $004750.5-730814$ & B0 & 12.55 & 12.56 & 0.07 & 0.28 \\
\hline 22 & 27 & 947 & $004751.6-731414$ & $\mathrm{~A} 2$ & 12.17 & 14.12 & 0.05 & $0.10^{c}$ \\
\hline 23 & 28 & 1662 & $004755.0-732114$ & B0 & 13.42 & 11.34 & 0.08 & 0.12 \\
\hline 24 & 31 & 361 & $00487.2-730632$ & B8: & 12.52 & 13.20 & 0.06 & 0.15 \\
\hline 25 & 32 & 1939 & $0048 \quad 10.2-724344$ & B1 & 14.20 & 10.95 & 0.03 & 0.00 \\
\hline 26 & 34 & -1 & $004818.7-732338$ & B2: & 13.81 & 0.00 & 0.00 & $-9.99^{\mathrm{b}}$ \\
\hline 27 & 35 & -1 & $004821.3-725102$ & B0: & 14.13 & 0.00 & 0.00 & $-9.99^{\mathrm{a}}$ \\
\hline 28 & 36 & 1934 & $004823.9-724338$ & B1 & 14.02 & 10.82 & 0.04 & 0.00 \\
\hline 29 & 37 & 1676 & $004827.0-730320$ & B5 & 12.88 & 11.43 & 0.07 & 0.03 \\
\hline 30 & 38 & 142 & $004828.2-730032$ & $\mathrm{~A} 0$ & 12.85 & 13.89 & 0.05 & $0.11^{\mathrm{c}}$ \\
\hline 31 & $39 \mathrm{~A}$ & 1476 & $004831.4-731532$ & W6 & 99.99 & 10.94 & 0.06 & $-9.99^{\mathrm{d}}$ \\
\hline 32 & 41 & 1767 & $004836.1-725250$ & B2 & 14.56 & 11.05 & 0.06 & -0.06 \\
\hline 33 & 42 & 2265 & $004840.2-725814$ & B5 & 13.49 & 12.16 & 0.09 & 0.03 \\
\hline 34 & 43 & 1875 & $004848.8-724614$ & B1 & 14.08 & 10.34 & 0.04 & -0.04 \\
\hline
\end{tabular}


Table 2-Continued

\begin{tabular}{|c|c|c|c|c|c|c|c|c|}
\hline \multirow[t]{2}{*}{$\#$} & \multirow{2}{*}{$\begin{array}{c}\mathrm{AV} \\
\#\end{array}$} & \multirow{2}{*}{$\begin{array}{l}\mathrm{UIT} \\
\#\end{array}$} & \multirow{2}{*}{$\begin{array}{l}\text { R.A. (2000.0) Dec } \\
\text { (Azzopardi and }\end{array}$} & \multirow{2}{*}{$\begin{array}{l}\text { Sp } \\
\text { Vigneau) }\end{array}$} & \multirow{2}{*}{$\mathrm{V}$} & \multicolumn{2}{|c|}{ UIT } & \multirow[t]{2}{*}{$\mathrm{E}(\mathrm{B}-\mathrm{V})$} \\
\hline & & & & & & $\mathrm{m}(162)$ & $\sigma$ & \\
\hline 35 & 45 & -1 & $004850.3-732202$ & $\mathrm{~B} 8$ & 14.15 & 0.00 & 0.00 & $-9.99^{\mathrm{a}}$ \\
\hline 36 & 48 & 1709 & $00493.4-732132$ & B3 & 11.03 & 9.45 & 0.12 & 0.06 \\
\hline 37 & 50 & 1765 & $004916.7-725232$ & B1 & 13.11 & 10.15 & 0.05 & 0.02 \\
\hline 38 & 51 & 1777 & $004932.8-725108$ & B0 & 14.05 & 10.81 & 0.03 & 0.03 \\
\hline 39 & 53 & 2495 & $004945.8-725238$ & $\mathrm{~A} 0$ & 12.96 & 14.41 & 0.05 & $0.14^{\mathrm{c}}$ \\
\hline 40 & 55 & 1197 & $004948.2-731745$ & B5 & 13.40 & 13.66 & 0.06 & 0.16 \\
\hline 41 & 56 & 1751 & $004951.7-725539$ & B5 & 11.17 & 9.14 & 0.04 & -0.02 \\
\hline 42 & 58 & 1769 & $004958.3-725145$ & $\mathrm{~B} 2$ & 14.38 & 10.83 & 0.04 & -0.06 \\
\hline 43 & 59 & 788 & $004958.4-731133$ & A0 & 13.40 & 13.54 & 0.06 & $0.04^{\mathrm{c}}$ \\
\hline 44 & 62 & 2361 & $00501.2-725503$ & B3 & 14.34 & 11.64 & 0.03 & -0.03 \\
\hline 45 & 63 & 672 & $00500.9-731008$ & $\mathrm{~A} 0$ & 13.48 & 13.12 & 0.06 & $0.00^{\mathrm{c}}$ \\
\hline 46 & 65 & 1448 & $00506.9-730739$ & B6 & 11.13 & 11.18 & 0.08 & 0.14 \\
\hline 47 & 66 & 1642 & $00506.7-731621$ & B0 & 13.48 & 11.27 & 0.09 & 0.11 \\
\hline 48 & 67 & 2039 & $005011.9-723227$ & B0 & 13.66 & 10.22 & 0.07 & 0.01 \\
\hline 49 & 69 & 2442 & $005018.0-725321$ & B0 & 13.35 & 10.01 & 0.21 & 0.02 \\
\hline 50 & 70 & 2007 & $005018.9-723803$ & B0 & 12.38 & 8.86 & 0.04 & 0.00 \\
\hline 51 & 73 & 1677 & $005028.4-730309$ & B0 & 14.08 & 10.95 & 0.07 & 0.04 \\
\hline 52 & 75 & 1757 & $005032.9-725227$ & O9 & 12.79 & 9.30 & 0.04 & 0.03 \\
\hline 53 & 77 & 1827 & $005034.2-724739$ & B0 & 13.91 & 10.41 & 0.03 & 0.01 \\
\hline 54 & 80 & 1824 & $005044.3-724733$ & B1 & 13.33 & 9.83 & 0.04 & -0.02 \\
\hline 55 & 82 & 1925 & $005049.8-724233$ & $\mathrm{~B} 2$ & 14.13 & 10.09 & 0.06 & -0.10 \\
\hline 56 & 83 & 1930 & $005052.8-724203$ & B1 & 13.38 & 9.80 & 0.04 & -0.03 \\
\hline 57 & 85 & 2440 & $00510.8-725257$ & B0 & 13.75 & 11.33 & 0.05 & 0.09 \\
\hline 58 & 87 & 3400 & $00518.3-724003$ & B0 & 13.90 & 12.76 & 0.11 & 0.19 \\
\hline 59 & 89 & 1121 & $005110.4-731545$ & B0 & 14.47 & 14.03 & 0.09 & 0.25 \\
\hline 60 & 90 & -1 & $0051 \quad 12.1-722809$ & A5 & 12.58 & 0.00 & 0.00 & $-9.99^{\mathrm{a}}$ \\
\hline 61 & 91 & 2325 & $005111.5-725509$ & $\mathrm{~B} 8$ & 12.61 & 12.36 & 0.04 & 0.08 \\
\hline 62 & 93 & 1940 & $005120.8-724121$ & B0: & 14.13 & 11.07 & 0.06 & 0.04 \\
\hline 63 & 94 & 1783 & $005120.6-724933$ & B1 & 13.99 & 10.15 & 0.04 & -0.05 \\
\hline 64 & 95 & 1885 & $005121.9-724403$ & O9 & 13.91 & 9.90 & 0.04 & -0.01 \\
\hline 65 & 99 & 1809 & $005125.4-724745$ & $\mathrm{~B} 2$ & 13.01 & 10.59 & 0.04 & 0.03 \\
\hline 66 & 100 & 1743 & $005126.9-725745$ & B3 & 14.29 & 11.44 & 0.05 & -0.04 \\
\hline 67 & 102 & 2036 & $005136.5-723209$ & $\mathrm{~B} 2$ & 14.29 & 11.11 & 0.07 & -0.03 \\
\hline 68 & 103 & 1847 & $005136.7-724557$ & B1 & 13.36 & 10.27 & 0.03 & 0.01 \\
\hline
\end{tabular}


Table 2-Continued

\begin{tabular}{|c|c|c|c|c|c|c|c|c|}
\hline \multirow[t]{2}{*}{$\#$} & \multirow{2}{*}{$\begin{array}{c}\mathrm{AV} \\
\#\end{array}$} & \multirow{2}{*}{$\begin{array}{l}\mathrm{UIT} \\
\#\end{array}$} & \multirow{2}{*}{$\begin{array}{l}\text { R.A. (2000.0) Dec } \\
\text { (Azzopardi and }\end{array}$} & \multirow{2}{*}{\multicolumn{2}{|c|}{$\begin{array}{l}\text { Sp } \\
\text { Iigneau) }\end{array}$}} & \multicolumn{2}{|c|}{ UIT } & \multirow[t]{2}{*}{$\mathrm{E}(\mathrm{B}-\mathrm{V})$} \\
\hline & & & & & & $\mathrm{m}(162)$ & $\sigma$ & \\
\hline 69 & 104 & 1800 & $005139.0-724757$ & B1 & 13.13 & 9.93 & 0.04 & 0.00 \\
\hline 70 & 105 & 3885 & $005141.9-722757$ & A0 & 12.24 & 12.61 & 0.07 & $0.05^{\mathrm{c}}$ \\
\hline 71 & 106 & 2005 & $005144.1-723715$ & B1 & 14.32 & 11.28 & 0.05 & 0.01 \\
\hline 72 & 109 & 1976 & $005150.6-723915$ & B1 & 13.73 & 10.43 & 0.03 & -0.01 \\
\hline 73 & 110 & 1877 & $005152.8-724403$ & A0 & 12.17 & 12.06 & 0.06 & $0.02^{\mathrm{c}}$ \\
\hline 74 & 111 & 2035 & $005156.7-723157$ & B1 & 13.84 & 10.52 & 0.06 & -0.01 \\
\hline 75 & 112 & 2031 & $005158.5-723315$ & B0 & 14.15 & 10.27 & 0.05 & -0.02 \\
\hline 76 & 114 & 1971 & $00523.1-723922$ & B0 & 14.93 & 11.10 & 0.05 & -0.02 \\
\hline 77 & 122 & 1932 & $005225.7-724052$ & B6 & 12.79 & 11.44 & 0.05 & 0.02 \\
\hline 78 & 123 & 1758 & $005227.8-725058$ & B8 & 13.22 & 11.59 & 0.06 & -0.03 \\
\hline 79 & 126 & 1961 & $005231.7-723922$ & B0 & 13.47 & 10.26 & 0.04 & 0.03 \\
\hline 80 & 131 & 1841 & $005241.0-724510$ & B8 & 12.61 & 11.94 & 0.07 & 0.05 \\
\hline 81 & 133 & 2003 & $005244.2-723646$ & B0 & 13.91 & 9.87 & 0.04 & -0.04 \\
\hline 82 & 137 & 1865 & $005253.0-724404$ & $\mathrm{~B} 4$ & 12.33 & 10.46 & 0.04 & 0.01 \\
\hline 83 & 138 & 1784 & $005252.9-724822$ & B0 & 14.28 & 10.94 & 0.05 & 0.02 \\
\hline 84 & 141 & 3723 & $005310.5-723340$ & B3: & 14.50 & 12.79 & 0.04 & 0.05 \\
\hline 85 & 143 & 1970 & $005327.1-723822$ & B0 & 14.12 & 10.32 & 0.04 & -0.02 \\
\hline 86 & 144 & 1742 & $005333.7-725616$ & B0 & 14.06 & 10.13 & 0.08 & -0.03 \\
\hline 87 & 145 & 1983 & $005336.0-723740$ & B1 & 13.35 & 10.10 & 0.05 & 0.00 \\
\hline 88 & 148 & 1882 & $005342.5-724228$ & B0 & 14.28 & 10.40 & 0.04 & -0.02 \\
\hline 89 & 149 & 1774 & $005353.6-724822$ & $\mathrm{~B} 2$ & 13.96 & 10.79 & 0.07 & -0.03 \\
\hline 90 & 150 & 4034 & $005358.3-722835$ & $\mathrm{~B} 6$ & 12.72 & 11.44 & 0.05 & 0.03 \\
\hline 91 & 151 & 1807 & $005359.6-724553$ & B5 & 12.30 & 10.27 & 0.06 & -0.02 \\
\hline 92 & 152 & 5913 & $00544.2-723140$ & A3 & 11.87 & 12.23 & 0.03 & $-0.06^{c}$ \\
\hline 93 & 153 & 3451 & $00544.0-723717$ & $\mathrm{~B} 8$ & 13.58 & 12.51 & 0.06 & 0.01 \\
\hline 94 & 154 & 3095 & $00549.9-724134$ & B0: & 13.55 & 13.30 & 0.05 & 0.26 \\
\hline 95 & 155 & 2975 & $0054 \quad 15.2-724259$ & B0: & 14.34 & 14.54 & 0.10 & 0.30 \\
\hline 96 & 156 & 4384 & $005419.4-721747$ & A0 & 14.17 & 13.99 & 0.06 & $0.01^{\mathrm{c}}$ \\
\hline 97 & 157 & 3938 & $005423.0-721705$ & B0 & 14.33 & 11.07 & 0.05 & 0.03 \\
\hline 98 & 158 & 3946 & $005423.5-721859$ & B1 & 14.06 & 10.22 & 0.04 & -0.05 \\
\hline 99 & 160 & 2340 & $005443.5-725153$ & B0 & 13.99 & 11.34 & 0.07 & 0.07 \\
\hline 100 & 164 & 4070 & $00 \begin{array}{lllll}55 & 16.0 & -72 & 41 & 17\end{array}$ & B0 & 14.16 & 10.71 & 0.04 & 0.01 \\
\hline 101 & 165 & 3994 & $005519.9-722559$ & B7 & 12.79 & 11.65 & 0.06 & 0.03 \\
\hline 102 & 169 & 4077 & $005540.3-724454$ & B1 & 13.90 & 11.07 & 0.05 & 0.03 \\
\hline
\end{tabular}


Table 2-Continued

\begin{tabular}{|c|c|c|c|c|c|c|c|c|}
\hline \multirow[t]{2}{*}{$\#$} & \multirow{2}{*}{$\begin{array}{l}\text { AV } \\
\#\end{array}$} & \multirow{2}{*}{$\begin{array}{c}\mathrm{UIT} \\
\#\end{array}$} & \multirow{2}{*}{$\begin{array}{l}\text { R.A. (2000.0) Dec } \\
\text { (Azzopardi and }\end{array}$} & \multirow{2}{*}{\multicolumn{2}{|c|}{$\operatorname{Sp}_{\text {Vigneau) }}$}} & \multicolumn{2}{|c|}{ UIT } & \multirow[t]{2}{*}{$\mathrm{E}(\mathrm{B}-\mathrm{V})$} \\
\hline & & & & & & $\mathrm{m}(162)$ & $\sigma$ & \\
\hline 103 & 171 & 6141 & $005548.2-723923$ & B6 & 13.34 & 12.77 & 0.05 & 0.09 \\
\hline 104 & 172 & 10309 & $005554.9-720854$ & $\mathrm{~B} 8$ & 13.37 & 13.37 & 0.06 & 0.10 \\
\hline 105 & 174 & 8802 & $005638.6-720136$ & A7 & 12.43 & 14.52 & 0.07 & $-0.06^{\mathrm{c}}$ \\
\hline 106 & 175 & 4036 & $005638.9-723630$ & B1 & 13.65 & 11.12 & 0.05 & 0.05 \\
\hline 107 & 176 & 3947 & $005639.7-722506$ & B1 & 13.93 & 11.10 & 0.04 & 0.03 \\
\hline 108 & 177 & 6454 & $005645.1-720330$ & $\mathrm{O} 5$ & 14.60 & 10.50 & 0.03 & 0.03 \\
\hline 109 & 178 & 3970 & $005648.6-722842$ & B2 & 14.38 & 11.29 & 0.06 & -0.03 \\
\hline 110 & 180 & 7161 & $005655.8-722412$ & B5 & 13.19 & 12.27 & 0.09 & 0.07 \\
\hline 111 & 181 & 11001 & $005657.8-721736$ & A0 & 13.83 & 13.15 & 0.04 & $-0.03^{c}$ \\
\hline 112 & 182 & 6480 & $00571.6-720806$ & B0 & 14.33 & 10.81 & 0.03 & 0.00 \\
\hline 113 & 184 & 7143 & $005714.4-722224$ & B8 & 14.18 & 12.57 & 0.07 & -0.03 \\
\hline 114 & 185 & 6777 & $005724.4-720130$ & B8: & 13.28 & 11.97 & 0.06 & 0.00 \\
\hline 115 & 186 & 4000 & $005727.8-723306$ & O9 & 14.10 & 10.63 & 0.05 & 0.03 \\
\hline 116 & 189 & 3958 & $005733.3-722848$ & B1 & 14.51 & 11.21 & 0.05 & -0.01 \\
\hline 117 & 190 & 11396 & $005735.1-722648$ & B6 & 13.52 & 13.19 & 0.08 & 0.11 \\
\hline 118 & 191 & 6482 & $005737.8-721300$ & B1 & 13.63 & 10.42 & 0.06 & 0.00 \\
\hline 119 & 192 & 6490 & $005738.2-722149$ & O9 & 14.58 & 11.06 & 0.05 & 0.03 \\
\hline 120 & 193 & 7152 & $005738.1-722431$ & B1 & 15.40 & 12.54 & 0.09 & 0.03 \\
\hline 121 & 194 & 4008 & $005745.6-723530$ & B0 & 13.95 & 10.73 & 0.04 & 0.03 \\
\hline 122 & 195 & 5818 & $005745.5-724012$ & B5 & 13.53 & 14.47 & 0.04 & 0.21 \\
\hline 123 & 196 & 7163 & $005754.7-722737$ & B1 & 13.93 & 11.57 & 0.09 & 0.07 \\
\hline 124 & 199 & 5274 & $00582.3-723537$ & A3 & 13.41 & 13.45 & 0.03 & $-0.09^{c}$ \\
\hline 125 & 200 & 4025 & $00588.1-723825$ & A0 & 12.10 & 11.37 & 0.05 & $-0.03^{c}$ \\
\hline 126 & 201 & 6479 & $005810.7-721055$ & B0 & 14.04 & 10.76 & 0.06 & 0.02 \\
\hline 127 & 202 & 6855 & $005815.5-720725$ & B0 & 14.33 & 11.20 & 0.05 & 0.04 \\
\hline 128 & 204 & 3998 & $005822.5-723513$ & B1: & 14.38 & 11.65 & 0.05 & 0.04 \\
\hline 129 & 205 & 11006 & $005823.5-722131$ & $\mathrm{~A} 2$ & 12.30 & 13.41 & 0.04 & $0.03^{\mathrm{c}}$ \\
\hline 130 & 207 & 6429 & $005833.7-715543$ & $\mathrm{O} 7$ & 14.37 & 10.70 & 0.06 & 0.05 \\
\hline 131 & 208 & 5549 & $005833.7-723925$ & O9 & 14.10 & 11.49 & 0.10 & 0.10 \\
\hline 132 & 209 & 7135 & $005835.9-722455$ & B0 & 14.46 & 11.45 & 0.04 & 0.05 \\
\hline 133 & 210 & 6484 & $005836.7-721619$ & B3 & 12.67 & 10.73 & 0.05 & 0.03 \\
\hline 134 & 211 & 7145 & $005841.8-722613$ & $\mathrm{~B} 8$ & 11.50 & 11.67 & 0.07 & 0.11 \\
\hline 135 & 213 & 6607 & $005855.1-715631$ & $\mathrm{~A} 2$ & 12.11 & 12.14 & 0.08 & $-0.05^{c}$ \\
\hline 136 & 214 & 6964 & $005855.2-721313$ & B3 & 13.34 & 12.15 & 0.03 & 0.09 \\
\hline
\end{tabular}


Table 2-Continued

\begin{tabular}{|c|c|c|c|c|c|c|c|c|}
\hline \multirow[t]{2}{*}{$\#$} & \multirow{2}{*}{$\begin{array}{c}\mathrm{AV} \\
\#\end{array}$} & \multirow{2}{*}{$\begin{array}{l}\text { UIT } \\
\#\end{array}$} & \multirow{2}{*}{$\begin{array}{l}\text { R.A. (2000.0) Dec } \\
\quad \text { (Azzopardi and }\end{array}$} & \multirow{2}{*}{$\begin{array}{l}\text { Sp } \\
\text { Vigneau) }\end{array}$} & \multirow{2}{*}{ V } & \multicolumn{2}{|c|}{ UIT } & \multirow[t]{2}{*}{$\mathrm{E}(\mathrm{B}-\mathrm{V})$} \\
\hline & & & & & & $\mathrm{m}(162)$ & $\sigma$ & \\
\hline 137 & 215 & 3955 & $005855.9-723201$ & B1 & 12.76 & 10.15 & 0.06 & 0.05 \\
\hline 138 & 217 & 7050 & $00592.3-72 \quad 1849$ & B2: & 14.59 & 11.48 & 0.03 & -0.03 \\
\hline 139 & 218 & 7064 & $00595.2-72 \quad 1937$ & B1 & 13.80 & 11.29 & 0.05 & 0.06 \\
\hline 140 & 219 & 7010 & $00597.7-72 \quad 1631$ & B2: & 14.50 & 11.23 & 0.06 & -0.04 \\
\hline 141 & 220 & 6446 & $005910.9-720543$ & O9 & 14.50 & 10.52 & 0.06 & -0.01 \\
\hline 142 & 221 & 3945 & $005910.2-723131$ & B0 & 13.46 & 11.34 & 0.05 & 0.12 \\
\hline 143 & 222 & 7080 & $005913.5-722055$ & B3 & 13.20 & 11.42 & 0.06 & 0.04 \\
\hline 144 & 223 & 4007 & $005913.6-723855$ & B0 & 13.67 & 10.64 & 0.02 & 0.04 \\
\hline 145 & 224 & 6732 & $005916.9-720437$ & B0 & 14.22 & 11.23 & 0.05 & 0.05 \\
\hline 146 & 225 & 4313 & $00 \quad 5916.8-722943$ & B8 & 14.05 & 13.07 & 0.03 & 0.02 \\
\hline 147 & 226 & 6481 & $005921.4-721707$ & B0 & 14.42 & 10.72 & 0.05 & -0.01 \\
\hline 148 & 229 & 8932 & $005927.5-720949$ & O9 & 11.86 & 10.98 & 0.16 & 0.24 \\
\hline 149 & 230 & 6435 & $005930.1-720102$ & B2 & 12.75 & 10.64 & 0.05 & 0.05 \\
\hline 150 & 231 & 10562 & 005929.6 -72 2014 & $\mathrm{~A} 0$ & 14.20 & 13.54 & 0.04 & $-0.03^{c}$ \\
\hline 151 & 232 & -1 & $005932.9-72 \quad 1043$ & O9 & 12.36 & 0.00 & 0.00 & $-9.99^{\mathrm{e}}$ \\
\hline 152 & 234 & 6440 & $005944.3-720413$ & B2 & 12.98 & 10.75 & 0.04 & 0.04 \\
\hline 153 & 237 & 7019 & $005953.5-721902$ & B8 & 12.59 & 12.26 & 0.03 & 0.07 \\
\hline 154 & 238 & 6475 & $005956.0-721332$ & O9 & 13.77 & 10.28 & 0.06 & 0.03 \\
\hline 155 & 240 & 3921 & $01001.1-722350$ & B3 & 14.03 & 11.42 & 0.05 & -0.02 \\
\hline 156 & 242 & 9303 & $01007.9-721356$ & B1 & 12.11 & 12.61 & 0.07 & 0.29 \\
\hline 157 & 245 & 6543 & $010016.7-715702$ & B5 & 13.34 & 11.74 & 0.06 & 0.01 \\
\hline 158 & 250 & 6589 & $010023.8-715926$ & B9 & 12.82 & 12.71 & 0.08 & $0.06^{\mathrm{c}}$ \\
\hline 159 & 251 & 3932 & $010022.9-723044$ & O7 & 14.75 & 11.09 & 0.06 & 0.05 \\
\hline 160 & 252 & 6450 & $010030.6-721056$ & B2: & 13.07 & 10.63 & 0.05 & 0.03 \\
\hline 161 & 257 & 6486 & $010045.1-722350$ & B3 & 12.79 & 10.87 & 0.05 & 0.03 \\
\hline 162 & 260 & 6453 & $010052.0-721332$ & B1 & 13.28 & 10.67 & 0.05 & 0.05 \\
\hline 163 & 263 & 6813 & $01016.9-721257$ & B6 & 12.85 & 12.07 & 0.04 & 0.07 \\
\hline 164 & 264 & 6422 & $01018.4-715956$ & B0 & 12.36 & 9.50 & 0.12 & 0.06 \\
\hline 165 & 266 & 6489 & $010110.1-722726$ & B1 & 12.63 & 9.90 & 0.06 & 0.04 \\
\hline 166 & 267 & 6439 & $\begin{array}{llllll}01 & 01 & 16.0 & -72 & 06 & 32\end{array}$ & O8 & 14.92 & 10.72 & 0.03 & -0.01 \\
\hline 167 & 268 & 6448 & $\begin{array}{llllll}01 & 01 & 16.4 & -72 & 12 & 39\end{array}$ & B0: & 13.14 & 10.42 & 0.06 & 0.07 \\
\hline 168 & 270 & 6477 & $0101 \quad 17.5-721727$ & B9 & 11.42 & 10.60 & 0.05 & $0.00^{\mathrm{c}}$ \\
\hline 169 & 271 & 6476 & $010120.5-721715$ & B0 & 13.46 & 10.59 & 0.06 & 0.06 \\
\hline 170 & 272 & 9881 & $010123.4-722009$ & B2 & 14.52 & 12.41 & 0.05 & 0.05 \\
\hline
\end{tabular}


Table 2-Continued

\begin{tabular}{|c|c|c|c|c|c|c|c|c|}
\hline \multirow[t]{2}{*}{$\#$} & \multirow{2}{*}{$\begin{array}{l}\text { AV } \\
\#\end{array}$} & \multirow{2}{*}{$\begin{array}{l}\mathrm{UIT} \\
\#\end{array}$} & \multirow{2}{*}{$\begin{array}{l}\text { R.A. (2000.0) Dec } \\
\text { (Azzopardi and }\end{array}$} & \multirow{2}{*}{\multicolumn{2}{|c|}{$\underset{\text { Jigneau) }}{\text { Sp V }}$}} & \multicolumn{2}{|c|}{ UIT } & \multirow[t]{2}{*}{$\mathrm{E}(\mathrm{B}-\mathrm{V})$} \\
\hline & & & & & & $\mathrm{m}(162)$ & $\sigma$ & \\
\hline 171 & 273 & 7855 & $010127.9-720703$ & A1 & 12.18 & 13.32 & 0.04 & $0.07^{\mathrm{c}}$ \\
\hline 172 & 274 & 6483 & $010129.8-722315$ & B0 & 14.02 & 10.60 & 0.06 & 0.01 \\
\hline 173 & 277 & 6824 & $010133.0-721439$ & B2 & 14.07 & 11.20 & 0.05 & -0.01 \\
\hline 174 & 279 & 6428 & $010135.2-720303$ & O9 & 14.20 & 10.46 & 0.04 & 0.01 \\
\hline 175 & 280 & 6580 & $010139.9-720227$ & B0 & 14.66 & 11.74 & 0.05 & 0.05 \\
\hline 176 & 282 & 6447 & $010150.4-721309$ & O7 & 14.83 & 10.81 & 0.04 & 0.02 \\
\hline 177 & 286 & 6605 & $010157.8-720415$ & A5 & 12.35 & 13.64 & 0.07 & $-0.06^{\mathrm{c}}$ \\
\hline 178 & 287 & 6444 & $010157.5-721239$ & B0 & 12.86 & 9.65 & 0.05 & 0.03 \\
\hline 179 & 290 & 6561 & $01020.8-720221$ & B1 & 13.93 & 11.49 & 0.07 & 0.06 \\
\hline 180 & 291 & 6816 & $01024.6-721527$ & B1 & 14.78 & 12.61 & 0.04 & 0.08 \\
\hline 181 & 292 & 6923 & $01024.5-721903$ & B3 & 13.10 & 11.52 & 0.05 & 0.06 \\
\hline 182 & 296 & 6445 & $01028.8-721315$ & O8 & 14.38 & 10.77 & 0.03 & 0.04 \\
\hline 183 & 297 & 6419 & $010210.4-720021$ & B7 & 12.18 & 10.63 & 0.06 & 0.00 \\
\hline 184 & 298 & 6560 & $\begin{array}{llllll}01 & 02 & 12.7 & -72 & 02 & 51\end{array}$ & A0 & 12.52 & 12.58 & 0.09 & $0.03^{\mathrm{c}}$ \\
\hline 185 & 299 & 6980 & $010213.9-722209$ & O9 & 14.61 & 12.34 & 0.07 & 0.13 \\
\hline 186 & 300 & 6442 & $010214.8-721115$ & B0 & 14.46 & 10.69 & 0.05 & -0.01 \\
\hline 187 & 301 & 6512 & $010215.7-715945$ & B0 & 14.24 & 11.24 & 0.08 & 0.05 \\
\hline 188 & 302 & 9841 & $010219.3-722203$ & B0 & 14.35 & 14.54 & 0.11 & 0.30 \\
\hline 189 & 303 & 6418 & $010222.3-720015$ & B1 & 12.81 & 10.16 & 0.07 & 0.05 \\
\hline 190 & 312 & 6441 & $010245.8-721203$ & B0 & 13.62 & 9.93 & 0.02 & -0.01 \\
\hline 191 & 314 & 8760 & $010248.6-721639$ & B2 & 12.90 & 10.57 & 0.12 & 0.04 \\
\hline 192 & 315 & 6438 & $010250.0-721009$ & B8 & 10.92 & 10.82 & 0.07 & 0.09 \\
\hline 193 & 318 & 6436 & $010254.8-720951$ & O9 & 13.59 & 10.53 & 0.05 & 0.06 \\
\hline 194 & 321 & 6433 & $010257.8-720803$ & O9 & 13.88 & 10.42 & 0.06 & 0.03 \\
\hline
\end{tabular}

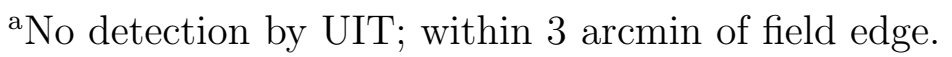

${ }^{\mathrm{b}}$ No detection by UIT; $\mathrm{m}(162)>14.5$.

${ }^{\mathrm{c}}$ Spectral type later than $\mathrm{B} 8$; intrinisic color and $\mathrm{E}(\mathrm{B}-\mathrm{V})$ therefore uncertain.

dNo magnitude supplied by AV.

eStar image is saturated on shortest UIT exposure. Convolution of UIT B5 bandpass with IUE spectrum SWP22007 gives $\mathrm{m}(162)=8.86$ 
Table 3. SMC HII Region Ha and FUV Fluxes

\begin{tabular}{|c|c|c|c|c|c|c|c|c|}
\hline \multirow[t]{2}{*}{ \# } & \multirow{2}{*}{$\begin{array}{c}\text { DEM } \\
\#\end{array}$} & \multicolumn{2}{|c|}{$\mathrm{KH} \mathrm{H} \alpha$} & \multicolumn{2}{|c|}{ FUV Stellar } & \multicolumn{2}{|c|}{ FUV Aperture } & \multirow{2}{*}{$\begin{array}{l}\text { "Sky" } \\
\text { Flux" }\end{array}$} \\
\hline & & Flux ${ }^{a}$ & $\sigma$ & Flux ${ }^{b}$ & $\sigma$ & Flux $^{b}$ & $\sigma$ & \\
\hline 1 & 14 & 5.00 & 1.00 & 1.047 & 0.081 & 2.397 & 0.074 & 0.022 \\
\hline 2 & 15 & 0.70 & 0.30 & 1.067 & 0.101 & 1.665 & 0.061 & 0.015 \\
\hline 3 & 17 & 1.70 & 0.20 & 0.190 & 0.016 & 0.559 & 0.036 & 0.006 \\
\hline 4 & 18 & 4.70 & 0.70 & 1.158 & 0.105 & 1.781 & 0.061 & 0.015 \\
\hline 5 & 20 & 2.90 & 0.50 & 0.755 & 0.048 & 1.987 & 0.063 & 0.015 \\
\hline 6 & 22 & 2.00 & 0.20 & 0.498 & 0.032 & 1.059 & 0.036 & 0.006 \\
\hline 7 & 23 & 8.40 & 1.00 & 0.253 & 0.015 & 1.017 & 0.041 & 0.006 \\
\hline 8 & 24 & 2.80 & 0.30 & 0.146 & 0.010 & 0.747 & 0.036 & 0.006 \\
\hline 9 & 30 & 2.90 & 0.30 & 0.405 & 0.033 & 1.217 & 0.037 & 0.006 \\
\hline 10 & 31 & 2.50 & 0.30 & 0.201 & 0.013 & 0.378 & 0.025 & 0.002 \\
\hline 11 & 32 & 40.00 & 4.00 & 6.202 & 0.463 & 11.814 & 0.107 & 0.040 \\
\hline 12 & 35 & 1.30 & 0.20 & 1.362 & 0.085 & 4.246 & 0.084 & 0.030 \\
\hline 13 & 36 & 0.90 & 0.20 & 1.793 & 0.108 & 4.655 & 0.085 & 0.030 \\
\hline 14 & 37 & 10.00 & 1.50 & 1.978 & 0.116 & 4.825 & 0.085 & 0.030 \\
\hline 15 & 40 & 4.20 & 0.50 & 0.779 & 0.050 & 1.446 & 0.037 & 0.006 \\
\hline 16 & 42 & 3.20 & 0.40 & 0.364 & 0.024 & 0.943 & 0.036 & 0.006 \\
\hline 17 & 43 & 6.50 & 0.70 & 0.545 & 0.032 & 1.177 & 0.037 & 0.006 \\
\hline 18 & 45 & 11.50 & 1.20 & 0.589 & 0.049 & 1.203 & 0.050 & 0.010 \\
\hline 19 & 46 & 2.30 & 0.30 & 0.720 & 0.033 & 2.779 & 0.034 & 0.022 \\
\hline 20 & 47 & 1.70 & 0.20 & 0.556 & 0.026 & 1.069 & 0.018 & 0.006 \\
\hline 21 & 49 & 5.00 & 1.30 & 1.669 & 0.114 & 3.286 & 0.061 & 0.015 \\
\hline 22 & 54 & 28.00 & 2.80 & 3.557 & 0.212 & 7.581 & 0.052 & 0.040 \\
\hline 23 & 55 & 25.00 & 2.50 & 4.585 & 0.217 & 8.356 & 0.047 & 0.040 \\
\hline 24 & 56 & 4.00 & 0.40 & 3.464 & 0.151 & 5.338 & 0.051 & 0.030 \\
\hline 25 & 57 & 0.70 & 0.20 & 0.312 & 0.020 & 0.745 & 0.018 & 0.006 \\
\hline 26 & 63 & 40.00 & 4.00 & 22.478 & 1.077 & 33.105 & 0.097 & 0.139 \\
\hline 27 & 69 & 19.50 & 4.00 & 4.353 & 0.217 & 13.957 & 0.059 & 0.075 \\
\hline 28 & 77 & 1.30 & 0.20 & 2.616 & 0.139 & 2.750 & 0.024 & 0.010 \\
\hline 29 & 80 & 13.50 & 1.50 & 5.744 & 0.326 & 8.266 & 0.044 & 0.089 \\
\hline 30 & 83 & 0.40 & 0.20 & 0.670 & 0.039 & 0.550 & 0.009 & 0.006 \\
\hline 31 & 84 & 0.20 & 0.20 & 0.379 & 0.024 & 0.276 & 0.006 & 0.002 \\
\hline 32 & 85 & 1.30 & 0.30 & 2.562 & 0.156 & 2.369 & 0.022 & 0.030 \\
\hline 33 & 86 & 1.30 & 0.30 & 2.145 & 0.126 & 2.465 & 0.022 & 0.030 \\
\hline 34 & 90 & 1.70 & 0.50 & 2.534 & 0.129 & 2.916 & 0.015 & 0.040 \\
\hline
\end{tabular}


Table 3-Continued

\begin{tabular}{|c|c|c|c|c|c|c|c|c|}
\hline \multirow[t]{2}{*}{$\#$} & \multirow{2}{*}{$\begin{array}{c}\text { DEM } \\
\#\end{array}$} & \multicolumn{2}{|c|}{$\mathrm{KH} \mathrm{H} \alpha$} & \multicolumn{2}{|c|}{ FUV Stellar } & \multicolumn{2}{|c|}{ FUV Aperture } & \multirow{2}{*}{$\begin{array}{l}\text { "Sky" } \\
\text { Flux }\end{array}$} \\
\hline & & Flux $^{\mathrm{a}}$ & $\sigma$ & Flux $^{b}$ & $\sigma$ & Flux $^{b}$ & $\sigma$ & \\
\hline 35 & 93 & 0.80 & 0.20 & 1.575 & 0.091 & 1.552 & 0.016 & 0.015 \\
\hline 36 & 94 & 1.30 & 0.20 & 1.603 & 0.080 & 1.653 & 0.016 & 0.015 \\
\hline 37 & 98 & 2.00 & 0.20 & 0.387 & 0.014 & 0.383 & 0.004 & 0.002 \\
\hline 38 & 100 & 0.70 & 0.20 & 0.299 & 0.017 & 0.353 & 0.005 & 0.006 \\
\hline 39 & 101 & 0.60 & 0.20 & 0.363 & 0.023 & 0.387 & 0.005 & 0.006 \\
\hline 40 & 102 & 3.40 & 0.40 & 1.410 & 0.070 & 1.251 & 0.009 & 0.010 \\
\hline 41 & 103 & 155.00 & 16.00 & 29.339 & 1.747 & 93.790 & 32.747 & 0.158 \\
\hline 42 & 111 & 1.20 & 0.20 & 0.521 & 0.037 & 0.491 & 0.006 & 0.006 \\
\hline
\end{tabular}

${ }^{\mathrm{a}} \times 10^{11} \mathrm{erg}\left(\mathrm{cm}^{2} \mathrm{~s}\right)^{-1}$

${ }^{\mathrm{b}} \times 10^{12} \operatorname{erg}\left(\mathrm{cm}^{2} \AA \mathrm{s}\right)^{-1}$

${ }^{\mathrm{c}} \times 10^{12} \mathrm{erg}\left(\mathrm{cm}^{2} \AA \mathrm{s}\right)^{-1}$. Estimated sky flux contributed by non-SMC background in the apertures, as described in the text. 
This figure "fig1.gif" is available in "gif" format from: http://arxiv.org/ps/astro-ph/9612152v1 
This figure "fig2a.gif" is available in "gif" format from: http://arxiv.org/ps/astro-ph/9612152v1 
This figure "fig2b.gif" is available in "gif" format from: http://arxiv.org/ps/astro-ph/9612152v1 
This figure "fig6.gif" is available in "gif" format from: http://arxiv.org/ps/astro-ph/9612152v1 